\section{Predictive factor and kappa angle analysis for visual satisfactions in patients with multifocal IOL implantation}

G Prakash, DR Prakash, A Agarwal, DA Kumar, A Agarwal and S Jacob

\begin{abstract}
Purpose To evaluate the visual acuity and quality-related satisfaction of patients implanted with a refractive design multifocal intraocular lens (IOL), and evaluate the factors predicting it including angle kappa.

Setting Dr Agarwal's Eye Hospital and Eye Research Centre, Chennai.

Methods In this prospective trial, 50 eyes of 44 consecutive patients were included. All patients underwent phacoemulsification with multifocal IOL implantation (Rezoom IOL, Abbott Medical Optics). The preoperative and postoperative assessment included slit lamp biomicroscopy, uncorrected visual acuity (UCVA), best-corrected visual acuity (BCVA) and kappa angle assessment. At 1 year, 37 patients (43 eyes), who finished follow-up, were asked to rate their symptoms on a graded questionnaire (0-5 for five queries).
\end{abstract}

Results The decimal scores for UCVA and BCVA were $0.38 \pm 0.21$ and $0.47 \pm 0.17$ (preoperative), and $0.75 \pm 0.22$ and $0.99 \pm 0.11$ (postoperative), respectively. Symptom scores were haloes $0.98 \pm 1.7$, glare $0.69 \pm 1.48$, blurred distance $1.0 \pm 1.7$, intermediate $1.34 \pm 1.6$, near $1.06 \pm 1.8$. On regression analysis haloes depended on angle kappa and distance UCVA $\left(R^{2}=0.26, P=0.029\right)$, and glare on angle kappa $\left(R^{2}=0.26, P=0.033\right)$. Poor satisfactions with distance, intermediate, and near vision were linked with distance UCVA $\left(R^{2}=0.17\right.$, $\left.P=2.3 \times 10^{-4}\right)$, distance UCVA $\left(R^{2}=0.1\right.$, $P=0.04)$, and near UCVA $\left(R^{2}=0.12, P=0.03\right)$, respectively. The strongest predictor, however, for overall visual discomfort was distance UCVA $\left(R^{2}=0.1, P=0.04\right)$.
Conclusions Our study suggests that there may be a role of misalignment between the visual and pupillary axis (angle kappa) in the occurrence of photic phenomenon after refractive multifocal IOL implantation. Eye (2011) 25, 1187-1193; doi:10.1038/eye.2011.150; published online 17 June 2011

Keywords: multifocal intraocular lens; visual satisfaction; kappa angle

\section{Introduction}

Multifocal intraocular lens (IOL) implantation for the correction of ametropia aims for a good unaided visual acuity for both, near and distance. This is done by creating multiple focal points, which focus for distance and near. ${ }^{1-3}$ Two inherent designs of multifocal optics in IOLs have been found that are used to develop these IOLs: refractive and diffractive. Refractive IOLs have multiple concentric rings in them with varying powers. Diffractive IOLs work on the Huygens-Fresnel principle. ${ }^{4-6}$ Both the lens designs have improved significantly from their earlier prototypes. These include aspheric optics, change in the size of rings and making the IOL dominant for distance or near, and modifying the anterior or posterior surface of the IOLs. ${ }^{3,5}$ Inspite of these modifications, the implantation of a multifocal IOL can have less than satisfactory visual acuity and quality, along with more photic phenomenon like haloes and glare compared with monofocal IOLs.

Researchers have noted these factors, and some studies have analyzed the governing factors for patient satisfaction after implantation of multifocal IOLs. ${ }^{7-21}$ Causes associated with
Dr Agarwal's Eye Hospital and Eye Research Centre, Chennai, India

Correspondence: A Agarwal, Dr Agarwal's Eye Hospital and Eye Research Centre, 19, Cathedral Road, Chennai 600086,

Tamilnadu, India Tel: + 91442811 6233; Fax: + 914428115871 . E-mail: dragarwal@ vsnl.com

Received: 8 October 2010 Accepted in revised form: 1 May 2011 Published online: 17 June 2011 
photic phenomena noted in previous studies have included IOL decentration, retained lens fragment, posterior capsular opacification, dry-eye syndrome, uncorrected visual acuity (UCVA), use of spectacles for distance purposes, postoperative astigmatism, and postoperative spherical equivalent. ${ }^{9,21}$ However, none of the studies in published literature have evaluated the role of misalignment between the visual axis and the pupillary axis, or the angle kappa, as a specific predictor for patient symptoms. The current study evaluates the visual acuity and quality-related satisfaction of patients implanted with a multifocal IOL, and evaluates the factors predicting it including angle kappa.

\section{Patients and methods}

This prospective trial was conducted at a tertiary care ophthalmic hospital. After a detailed explanation of the procedure, the merits and demerits of a refractive multifocal IOL, a written informed consent was obtained from all patients. A total of 50 eyes of 44 consecutive patients were recruited in this prospective trial. All patients were scheduled for elective cataract surgery and had no other ocular pathology. Each eye was considered as a single case and all examinations in the study were done monocularly.

Preoperative exclusion criteria for the study included monofocal IOL in the fellow eye, more than $1 \mathrm{D}$ of astigmatism before surgery, retinal pathology, any corneal opacity or a history of diabetes mellitus, unrealistic expectations, professional or habitual night driving.

The preoperative assessment included slit lamp biomicroscopy, UCVA, best-corrected visual acuity (BCVA), refraction, Orbscan corneal analysis (Bausch and Lomb, Rochester, NY, USA), kappa angle assessment (Orbscan anterior segment analysis system, Bausch and Lomb), and dilated retinal examination. (Kappa angle measurement by Orbscan has been found to be significantly correlated to synoptophoric measurement, even though the values are higher with the former. ${ }^{22}$ It has also been used in a large population-based survey to measure kappa angle. ${ }^{23}$ )

Near-vision assessment was done on the $N$-type notation, which is a reduced Snellen's type notation. Higher values mean worse visual acuity in this scenario, as is with the denominator of Snellen's notation. This inadvertently caused the confusion in the charts. The approximate font sizes are as follows: $\mathrm{N} 48 \rightarrow 20 \mathrm{~mm}, \mathrm{~N} 36 \rightarrow 10 \mathrm{~mm}, \mathrm{~N} 24 \rightarrow 5 \mathrm{~mm}$, $\mathrm{N} 12 \rightarrow 4 \mathrm{~mm}, \mathrm{~N} 10 \rightarrow 3 \mathrm{~mm}, \mathrm{~N} 8 \rightarrow 2 \mathrm{~mm}, \mathrm{~N} 6 \rightarrow 1 \mathrm{~mm}$.

All cases were targeted for emmetropia. All patients included in the study had a refractive multifocal lens (Rezoom Acrylic Multifocal lens, Abott Medical Optics, Santa Anna, CA, USA) The Rezoom Multifocal is a posterior chamber IOL having concentric rings with five focusing zones for distance and near according to the illumination conditions.

\section{Surgical technique}

All surgeries were done by a single surgeon (Am A), well experienced in phacoemulsification. A topographyguided clear corneal incision was made with a $2.8-\mathrm{mm}$ keratome (Sharpedge Slimline, Ahmedabad, India) in the phacoemulsification group. The anterior chamber was deepened with 2\% hydroxyl propyl methyl cellulose (Viscomet, Sun Pharmaceuticals, Mumbai, India) and a continuous curvilinear anterior capsulorhexis was performed. Phacoemulsification was done using the phaco chop technique and the IOL was implanted. None of the patients required sutures to secure the wound. Intraoperative exclusion criteria were determined before the commencement of the study. They were: iris trauma, posterior capsular rupture with or without vitreous loss, and inability to place the IOL in the capsular bag. Two cases (one iris trauma, one posterior capsular rupture) were excluded from the study using these criteria. The postoperative follow-up was done as per the standard protocol of the institution. Postoperative topical medicines were started as follows: ofloxacin $0.3 \%$ four times a day, prednisolone phosphate four times a day and tropicamide $0.8 \%$ plus phenylephrine $5 \%$ thrice a day in all patients, and timolol maleate $0.5 \%$ twice a day whenever required. After the first week, all medications were stopped except the steroid and antibiotic, which were subsequently tapered over 1 month. Five cases were lost to follow-up ( $10 \%$ cases) before completion of 12 months. After completion of 1-year follow-up, the patients were asked to rate their symptoms. Therefore, a total of 43 eyes (37 cases) completed 1-year follow-up.

\section{Questionnaire}

The questionnaire was built using a survey of 25 unsatisfied patients with multifocal implantation. They were asked to write down their major symptoms. Then this questionnaire was created based on the responses. It was then given in a pilot comparison with 10 satisfied and 10 unsatisfied patients. The mean scores of the two groups were 'satisfied' $0.2 \pm 0.4$ vs 'unsatisfied' $7.3 \pm 4.1$ $(P<0.001$, rank sum test), validating the discriminating ability of score. The questionnaire was filled after 12-months follow-up to wait for any possible neuroadaptation as has been suggested by certain authors. ${ }^{3,6}$ The patients were asked to rate their overall satisfaction as excellent (0), very good (1), good (2), fair (3), poor (4), very poor (5) for their visual acuity related symptoms. Zero was considered to be 'no requirement of glasses', 3 meant use of glasses sometimes when doing 
certain work (for example, reading fine print, driving), and 5 meant use of refractive correction for all waking hours for all professional and personal activities.

For photic phenomenon, a grade of 0 meant no symptoms at all 140, grade 1: inconsistent symptoms, not bothersome; grade 2: symptoms mildly bothersome in certain situations only (for example, night driving) and can carry out activity without problem; grade 3: symptoms moderately bothersome in certain situations only, may need extra effort for activity; grade 4: either severely bothersome in certain situations or moderately bothersome in all situations; and grade 5: severely bothersome in all situations. For the photic phenomenon mild botheration meant that the patient could do his/her work without any extra effort, for example, may continue to drive, being aware of the glare/halo. Moderate botheration meant increased level of attention or change in activity pattern, for example, driving slow and carefully. Severe botheration meant cessation/avoidance of that activity, for example, the patient had stopped night driving because of symptoms. The questionnaire was explained to the patient in detail before asking them to fill in the responses. The symptoms assessed were difficulty in distance, intermediate and near vision, and photic phenomenon assessed were haloes and glare with maximum worse score of 5 each in all the five categories, with a total possible score of 25 . We certify that all applicable institutional and governmental regulations concerning the ethical use of human volunteers were followed during this research.

\section{Results}

\section{Demographics}

Those patients who finished the follow-up were included in the analysis. The mean age of the patients at the time of surgery was $59 \pm 12.3$ years. There were 20 males and 17 females. The mean angle kappa was $4.9 \pm 1.5^{\circ}$ (range 2.3 to $7.6^{\circ}$ ).

\section{Visual outcomes}

The visual acuities are represented in decimal scores. The preoperative UCVA and BCVA were $0.38 \pm 0.21$ and $0.47 \pm 0.17$, respectively. The postoperative UCVA and BCVA were $0.75 \pm 0.22$ and $0.99 \pm 0.11$, respectively, at the time of evaluation. The postoperative refractive error was $-0.22 \pm 0.53 \mathrm{D}$ Sphere (ranging from $-1 \mathrm{D}$ to $+1 \mathrm{D}$ ) and $-0.34 \pm 0.57 \mathrm{D}$ cylinder (ranging from -1.75 to $0 \mathrm{D}$ ). The mean postoperative spherical equivalent was $-0.38 \pm 0.68 \mathrm{D}$ (ranging from -2 to $0.50 \mathrm{D}$ ).

The preoperative uncorrected near vision was $\mathrm{N}$ $14.8 \pm 6.3$ and best-corrected near vision was $N$ 9.7 \pm 5.2 .
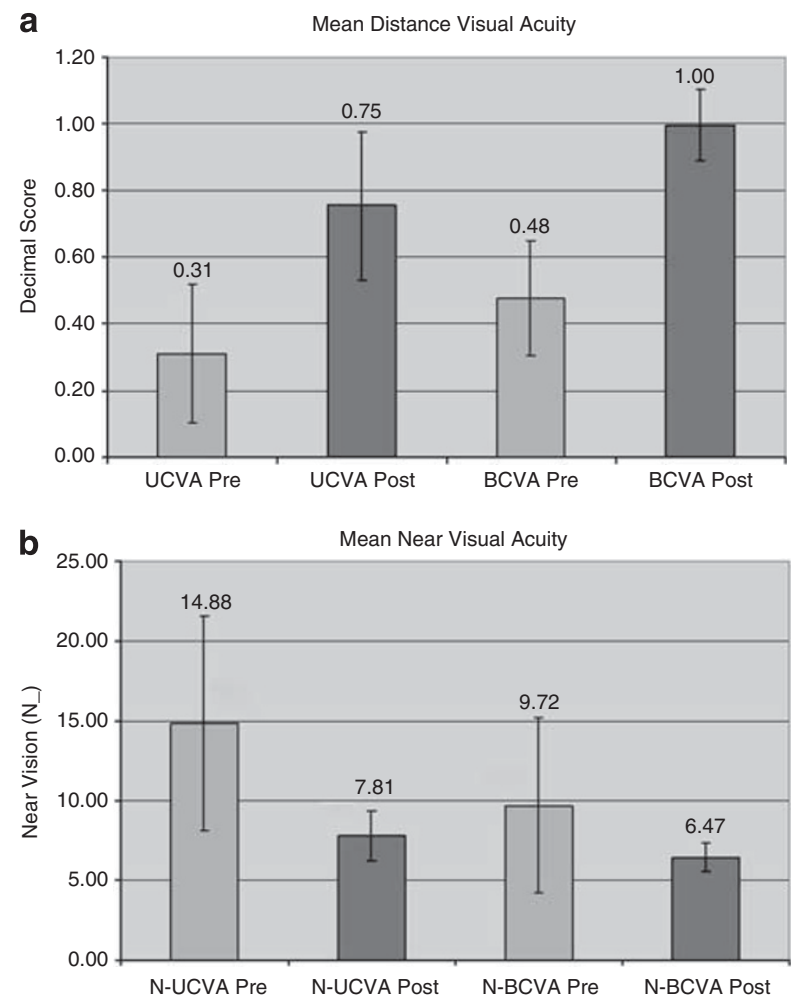

Figure 1 (a) Mean and one standard deviation outcomes for pre and postoperative distance-uncorrected and BCVA in the decimal score. The decimal score is derived by computing the numerical value of the Snellen's fraction; hence, a lower value on the bar chart denotes a lower visual acuity. (b) Mean and one standard deviation outcomes for pre and postoperative near uncorrected and BCVA in N-type near-vision values. As there is no fraction here, the raw values are provided and a lower value on the bar chart denotes a higher visual acuity.

The postoperative uncorrected near vision was $\mathrm{N}$ $7.8 \pm 1.6$ and best-corrected near vision was N $6.4 \pm 0.85$.

All the postoperative visual acuity parameters were significantly better than the preoperative counterparts $(P<0.05$, paired $t$-tests) (Figures $1 \mathrm{a}$ and $\mathrm{b})$. An uncorrected monocular distance vision $\geq 20 / 32$ was seen in 34 cases $(79.1 \%)$, and an uncorrected monocular near vision $\geq \mathrm{N} 8$ was seen in 32 cases $(74.4 \%)$.

\section{Symptom scores and parameters}

The mean symptom scores were $0.98 \pm 1.7$ for haloes (out of 5), $0.69 \pm 1.48$ for glare (out of 5 ), $1.0 \pm 1.7$ for blurred distance (out of 5), $1.34 \pm 1.6$ for blurred intermediate (out of 5), $1.06 \pm 1.8$ for blurred near (out of 5), and $5.3 \pm 6.8$ as overall score (out of 25). Excluding patients who had no complaints, the score in patients who were even mildly not satisfied was $8.2 \pm 5.4$. Figure 2 demonstrates a graphical representation and the mean scores of the overall cohort and only of those not fully satisfied from multifocal IOL implantation. 


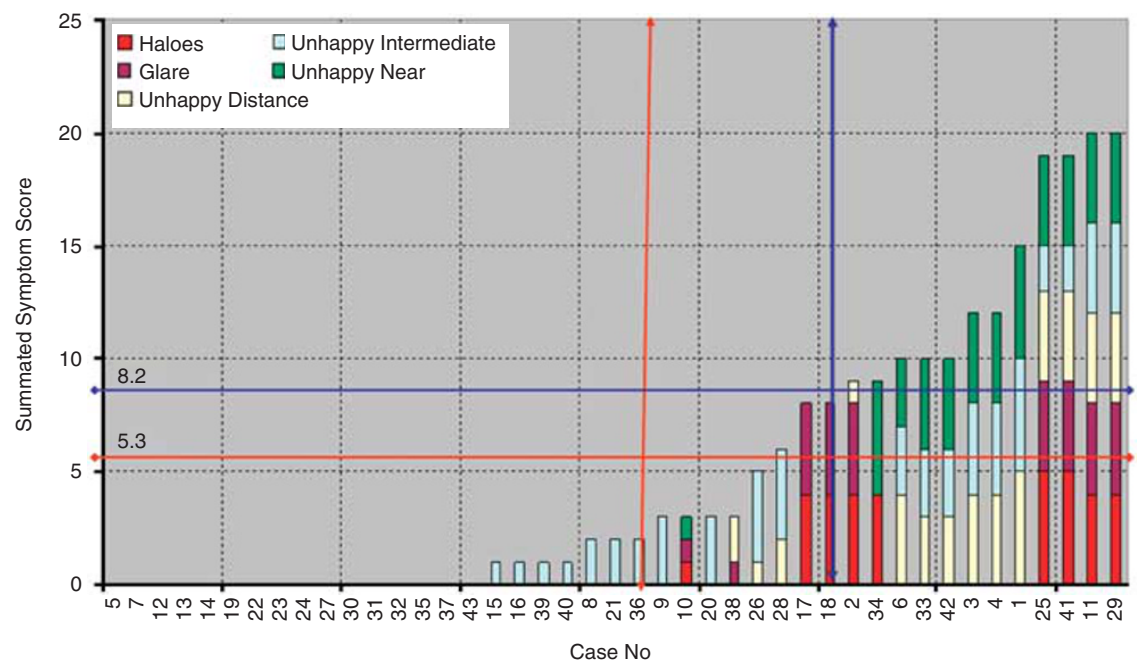

Figure 2 Bar chart showing summated symptom score of all the patients arranged according to increasing symptom score. The vertical lines show $50 \%$ of data and horizontal lines show mean value. The red lines are for overall (asymptomatic and symptomatic patients) and the blue lines are for only symptomatic patients.

A total of $31(72.1 \%)$ cases had no reportable haloes and $34(79.06 \%)$ cases had no complaints of glare. A total of $29(67.4 \%)$ cases had no problems with distance vision, $22(51.1 \%)$ cases had no problems with intermediate vision, and $31(72.1 \%)$ patients had no problems with near vision. The mean score was 5 .

\section{Correlation between symptom scores and parameters}

The analyzed factors considered to be responsible were angle kappa, gender, age, and postoperative parameters of distance UCVA, near UCVA, spherical error, cylindrical power, spherical equivalent, distance BCVA, and near BCVA. Regression analysis was performed to find out the association between the symptoms and factors responsible. It was seen that the perceived severity of haloes depended on angle kappa and distance UCVA $\left(R^{2}=0.26, P=0.029\right)$, and glare depended on angle kappa $\left(R^{2}=0.26, P=0.033\right)$ alone (Figures $3 \mathrm{a}$ and $\mathrm{b}$ ). The symptoms of poor satisfaction with distance vision, intermediate vision, and near vision were linked with distance UCVA $\left(R^{2}=0.17, P=2.3 \times 10^{-4}\right)$, distance UCVA $\left(R^{2}=0.1, P=0.04\right)$, and blurred near $=$ near UCVA $\left(R^{2}=0.12, P=0.03\right)$, respectively. The strongest predictor, however, for overall visual discomfort was distance UCVA $\left(R^{2}=0.1, P=0.04\right)$.

\section{Discussion}

Multifocal IOL designs have come a long way since the earlier prototypes. At the cost of mild reduction in contrast sensitivity, many patients are satisfied with these newer models. ${ }^{3,5,6,24}$ Neuro-adaptation may also have a
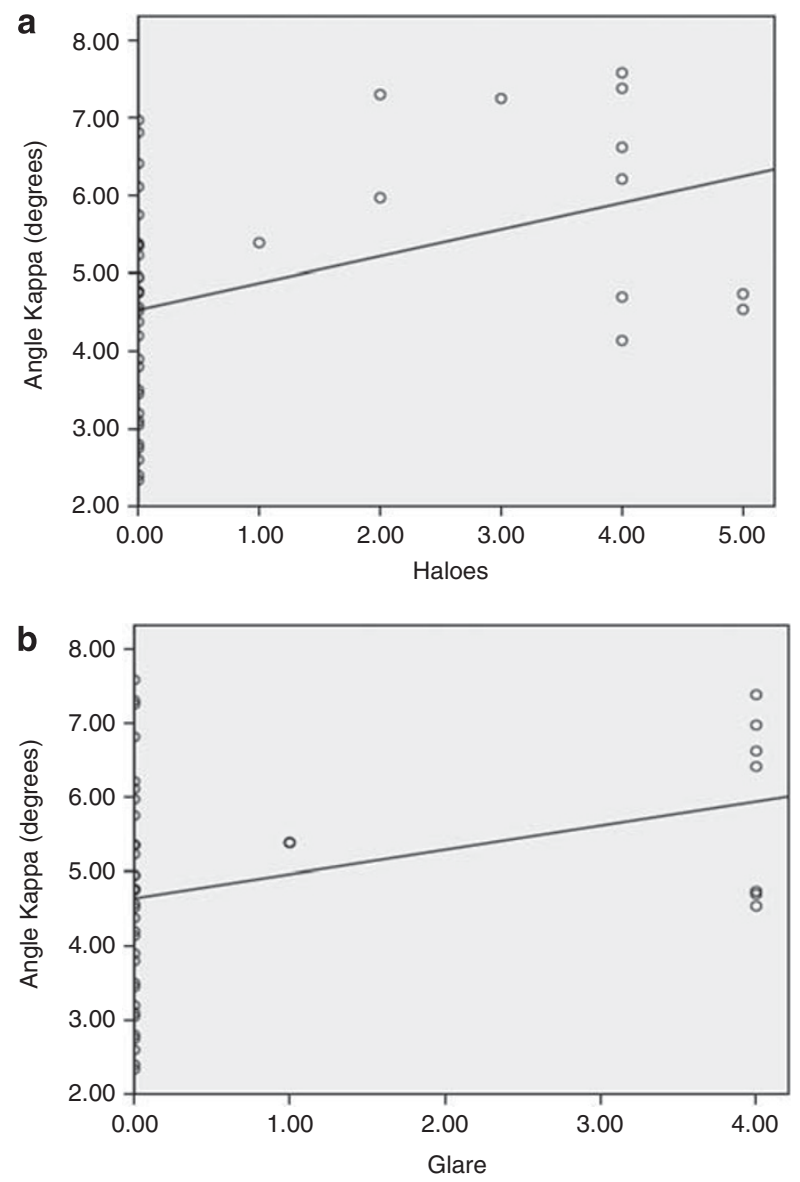

Figure 3 (a) Regression plot between the photic phenomenon of haloes ( $x$ axis) and the kappa angle ( $y$ axis). (b) Regression plot between the photic phenomenon of glare ( $x$ axis) and the kappa angle ( $y$ axis). 
major role in some cases and hence enough time should be provided before drawing a conclusion on the severity of photic phenomenon. Improved patient compliance noticed with these newer IOLs was seen in the current study too. The distance and near visual outcome was satisfactory in a large number of cases. We found that in cases having dissatisfaction, uncorrected postoperative visual acuity was the most important factor for patient satisfaction. This is intuitive because low intensity photic symptoms would occur in certain conditions, like around light sources or in night time driving, however, the effect of poor UCVA stays for the patient in all waking hours. Other than these factors, there was no effect of any other factor in the final resolution acuity satisfaction for distance or near. We did not find that cylindrical power had an effect independent of UCVA in the patient dissatisfaction. Walcow and Klemen had findings similar to that seen by us with UCVA in a questionnaire-based study on a diffractive IOL, however, they found cylindrical power to be an additionally important independent predictor of dissatisfaction. ${ }^{21}$ The reason for this difference can be in our exclusion of high cylinders from the preoperative data and the low postoperative cylinders. We agree that high cylinders and induced higher order aberration (acquired aberropia) may have an impact on the final satisfaction of the patients. ${ }^{25,26}$ We did not perform a wavefront analysis in our patients. A study correlating visual symptoms, angle kappa (and therefore coma aberration, which may be linked) and other higher order aberrations may provide further information on the same. ${ }^{27-29}$

Both the photic phenomenon evaluated in the study, that is, haloes and glare, were found to have an association with angle kappa, which represents the angle between the visual axis and the pupillary axis. Even though many patients with high angle kappa were also asymptomatic, the strength of association was statistically significant, suggesting that angle kappa values may be considered in preoperative decision making in cases of multifocal IOL implantation. The reason for this association needs to be evaluated in detail, with simulation methods perhaps like ray tracing to confirm whether edge effect from the anterior IOL surface's rings may be responsible for the same. A higher angle kappa means that a fovea-centric ray would pass closer to the edge of the IOL rings and not via the pupillary area exactly concentric and ahead of the centre of the IOL (Figures $4 \mathrm{a}$ and $\mathrm{b}$ ). However, the IOL centre may be dependent on the centre of the capsular bag even in a 'well-centered' implantation that may result in an additional variable, which perhaps further studies will look into.

A detailed discussion on the accommodative IOLs is out of the scope of the current manuscript that primarily deals with the visual effects of multifocal IOL. It remains
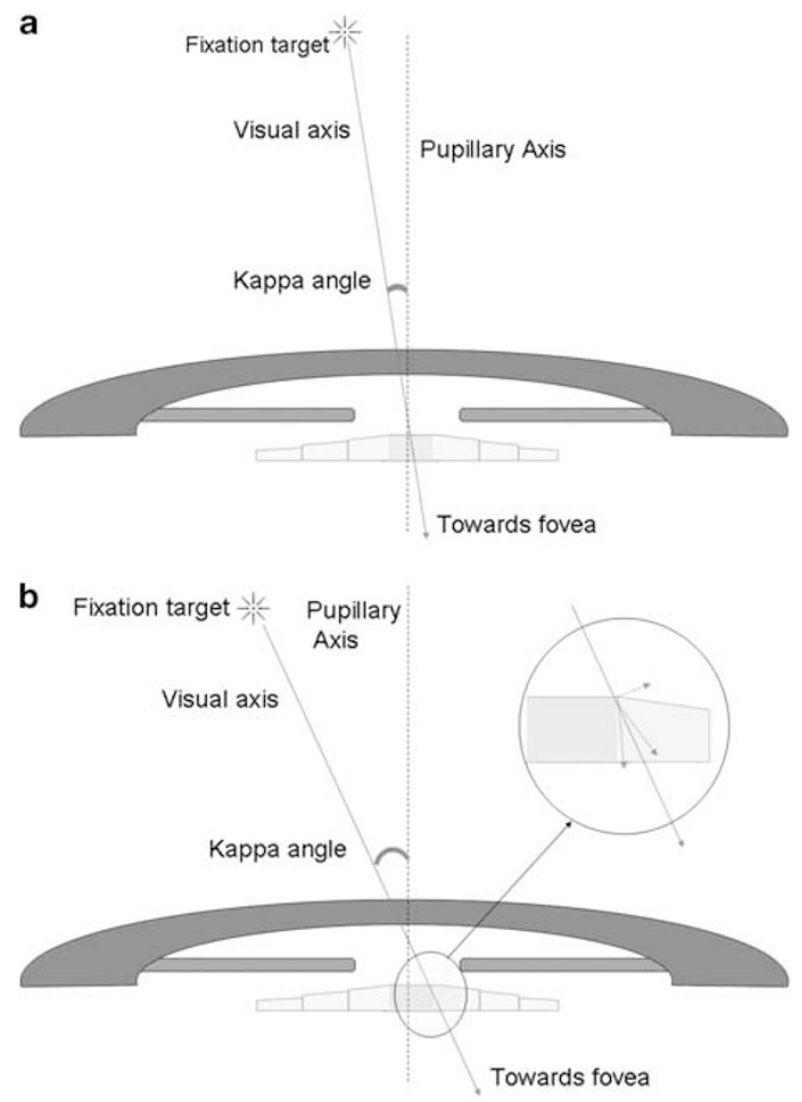

Figure 4 (a) Schematic ray diagram showing the incident ray passing through the central area in an eye with small angle kappa. (b) Schematic ray diagram showing the incident ray passing through the ring's edge area in an eye with large angle kappa.

to be seen whether kappa angle would have a similar effect on the optics of an accommodative IOL.

One of our patients had a spherical equivalent of $-2 \mathrm{D}$, therefore, ruling out any benefit of the multifocal effect. However, the aim of the study was to analyze the effects of multifocal IOL implantation on the perceived visual acuity, even after spectacle correction, and thus we did not rule out patients on the postoperative refraction. Never the less, this can be a possible exclusion criteria for another study looking at the optics and range of multifocality induced by these IOLs.

Each eye was defined as a single case and all these patients were asked for the symptoms uniocularly, and were marked on the questionnaire differently. The grade and details of symptoms were different for both eyes in many of our bilateral cases. This, in a way, validates the fact that the symptoms were due to surgery and not due to patient 'psyche'. We avoided using symptom analysis in bilateral cases as a 'single patient' to rule out the masking effects of binocular vision/summation over the symptoms. It may be noted that most self-reporting questionnaires are biased by the expectation levels and 
the mindset of the patient itself, and therefore, in spite of a scale based on objective parameters, the subjective perception of a symptom may vary from one patient to another. It needs to be seen whether the lens can be customized to match the kappa angle of the patient. Because of multiple variable factors including capsular contraction, memory of the haptics and IOL rotation, it seems unlikely that a multifocal IOL intentionally decentered towards the visual axis would stay in the same position in the postoperative period. Donnenfield and Holladay have performed pupilloplasty to centre the pupil and improve the waxy vision in certain such cases with high angle kappa. ${ }^{30}$ However, the IOL can itself be adjusted in a case of glued IOL for aphakia. ${ }^{31}$ In cases with glued multifocal IOL where the IOL is being placed without capsular support, one of the haptics may be pulled furthermore to position the central ring of IOL under the visual axis before tucking the haptic to fixate the IOL. A feasibility study for the same with glued IOL is currently undergoing in our institution and the results may throw more light on this evolving concept.

The perception of photic phenomenon is multifactorial as evaluated in previous studies. ${ }^{9,21}$ Our study suggests that there may be an additional role of misalignment between the visual and pupillary axis in the occurrence of photic phenomenon after multifocal IOL implantation. Further studies will be required to analyze the effect of the same on induced higher order aberrations, and contrast sensitivity after multifocal IOL implantation.

\section{Summary}

\section{What was known before}

- Many patients with multifocal IOL can be unhappy after implantation due to visual symptoms.

What this study adds

- This study analyzes the predictive factors and look at kappa angle as one of the reasons for visual complaints.

\section{Conflict of interest}

Dr Amar Agarwal is a paid consultant for Abbott Medical Optics and Bausch and Lomb. None of the other authors has a financial or propriety interest in any of the products and methods in the manuscript, or lack thereof.

\section{References}

1 Bellucci R. Multifocal intraocular lenses. Curr Opin Ophthalmol 2005; 16: 33-37.

2 Lane SS, Morris M, Nordan L, Packer M, Tarantino N, Wallace III RB. Multifocal intraocular lenses. Ophthalmol Clin North Am 2006; 19: 89-105.

3 Buznego C, Trattler WB. Presbyopia-correcting intraocular lenses. Curr Opin Ophthalmol 2009; 20: 13-18.
4 Jay JL, Chakrabarti HS, Morrison JD. Quality of vision through diffractive bifocal intraocular lens. Br J Ophthalmol 1991; 75: 359-366.

5 Cillino S, Casuccio A, Di Pace F, Morreale R, Pillitteri F, Cillino G, Lodato G. One-year outcomes with newgeneration multifocal intraocular lenses. Ophthalmology 2008; 115: 1508-1516.

6 Palomino Bautista C, Carmona González D, Castillo Gómez A, Bescos JA. Evolution of visual performance in 250 eyes implanted with the TecnisZM900 multifocal IOL. Eur J Ophthalmol 2009; 19: 762-768.

7 Häring G, Dick HB, Krummenauer F, Weissmantel U, Kröncke W. Subjective photic phenomena with refractive multifocal and monofocal intraocular lenses.results of a multicenter questionnaire. J Cataract Refract Surg 2001; 27: 245-249.

8 Dick HB, Krummenauer F, Schwenn O, Krist R, Pfeiffer N. Objective and subjective evaluation of photic phenomena after monofocal and multifocalintraocular lens implantation. Ophthalmology 1999; 106: 1878-1886.

9 Woodward MA, Randleman JB, Stulting RD. Dissatisfaction after multifocal intraocular lens implantation. J Cataract Refract Surg 2009; 3: 992-997.

10 Pepose JS. Maximizing satisfaction with presbyopiacorrecting intraocular lenses: the missing links. Am J Ophthalmol 2008; 146: 641-648.

11 Kohnen T, Kook D, Auffarth GU, Derhartunian V. Use of multifocal intraocular lenses and criteria for patient selection. Ophthalmology 2008; 105: 527-532.

12 Chang DF. Prospective functional and clinical comparison of bilateral ReZoom and ReSTOR intraocular lenses in patients 70 years or younger. J Cataract Refract Surg 2008; 34: 934-941.

13 Blaylock JF, Si Z, Aitchison S, Prescott C. Visual function and change in quality of life after bilateral refractive lens exchange with the ReSTOR multifocal intraocular lens. J Refract Surg 2008; 24: 265-273.

14 Chiam PJ, Chan JH, Haider SI, Karia N, Kasaby H, Aggarwal RK. Functional vision with bilateral ReZoom and ReSTOR intraocular lenses 6 months after cataract surgery. J Cataract Refract Surg 2007; 33: 2057-2061.

15 Alfonso JF, Fernández-Vega L, Baamonde MB, Montés-Micó R. Prospective visual evaluation of apodized diffractive intraocular lenses. J Cataract Refract Surg 2007; 33: 1235-1243.

16 Mester U, Hunold W, Wesendahl T, Kaymak H. Functional outcomes after implantation of Tecnis ZM900 and Array SA40 multifocal intraocular lenses. J Cataract Refract Surg 2007; 33: 1033-1040.

17 Pepose JS, Qazi MA, Davies J, Doane JF, Loden JC, Sivalingham V, Mahmoud AM. Visual performance of patients with bilateral vs combination Crystalens, ReZoom, and ReSTOR intraocular lens implants. Am J Ophthalmol 2007; 144: 347-357.

18 Chiam PJ, Chan JH, Aggarwal RK, Kasaby S. ReSTOR intraocular lens implantation in cataract surgery: quality of vision. J Cataract Refract Surg 2006; 32: 1459-1463.

19 Sen HN, Sarikkola AU, Uusitalo RJ, Laatikainen L. Quality of vision after AMO Array multifocal intraocular lens implantation. J Cataract Refract Surg 2004; 30: 2483-2493.

20 Hunkeler JD, Coffman TM, Paugh J, Lang A, Smith P, Tarantino N. Characterization of visual phenomena with the 
array multifocal intraocular lens. J Cataract Refract Surg 2002; 28: 1195-1204.

21 Walkow L, Klemen UM. Patient satisfaction after implantation of diffractive designed multifocal intraocular lenses in dependence on objective parameters. Graefes Arch Clin Exp Ophthalmol 2001; 239: 683-687.

22 Basmak H, Sahin A, Yildirim N, Papakostas TD, Kanellopoulos AJ. Measurement of angle kappa with synoptophore and Orbscan II in a normal population. J Refract Surg 2007; 23: 456-460.

23 Hashemi H, KhabazKhoob M, Yazdani K, Mehravaran S, Jafarzadehpur E, Fotouhi A. Distribution of angle kappa measurements with Orbscan II in a population-based survey. J Refract Surg 2010; 26: 966-971.

24 Leyland M, Zinicola E. Multifocal versus monofocal intraocular lenses in cataract surgery: a systematic review. Ophthalmology 2003; 110: 1789-1798.

25 Agarwal A, Prakash G, Jacob S, Ashokkumar D, Agarwal A. Can uncompensated higher order aberration profile, or aberropia be responsible for subnormal best corrected vision and pseudo-amblyopia. Med Hypotheses 2009; 72 574-577.
26 Agarwal A, Jacob S, Agarwal A. Aberropia: a new refractive entity. J Cataract Refract Surg 2007; 33: 1835-1836.

27 Espinosa J, Mas D, Kasprzak HT. Corneal primary aberrations compensation by oblique light incidence. J Biomed Opt 2009; 14: 044003.

28 Lu F, Wu J, Shen Y, Qu J, Wang Q, Xu C, Chen S, Zhou X, He JC. On the compensation of horizontal coma aberrations in young human eyes. Ophthalmic Physiol Opt 2008; 28: $277-282$.

29 Tabernero J, Benito A, Alcón E, Artal P. Mechanism of compensation of aberrations in the human eye. J Opt Soc Am A Opt Image Sci Vis 2007; 24(10): 3274-3283.

30 Chang DF, Claoué C, Donnenfeld ED, Hardten DR, Knorz M, Pepose JS et al. Annual IOL Issue: IOL options in 2008. Cataract and Refractive Surgery Today. January/February 2008. http://www.crstodayeurope.com/Issues/0108/ crste0108_12.pdf (accessed 12 August 2010).

31 Agarwal A, Kumar DA, Jacob S, Baid C, Agarwal A, Srinivasan S. Fibrin glue assisted sutureless posterior chamber intraocular lens implantation in eyes with deficient posterior capsules. J Cataract Refract Surg 2008; 34: 1433-1438. 